\begin{tabular}{|c|c|c|}
\hline & & \\
\hline & $\begin{array}{l}\text { de vache } \\
\text { (Söldner) }\end{array}$ & $\begin{array}{l}\text { de femme } \\
\text { (Laxa) }\end{array}$ \\
\hline$\ldots \ldots \ldots \ldots \ldots \ldots \ldots \ldots$ & 10.62 & 22.2 \\
\hline Chlorure de potassium . ................. & 9.16 & 10.4 \\
\hline Phosphate de potassium . ................ & 21.99 & 17.8 \\
\hline Sulfate de potassium . . . . . . . . . . . . . . . . & - & 0.7 \\
\hline 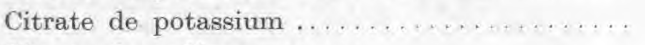 & 5.47 & 14.0 \\
\hline Citrate de sodium . . . . . . . . . . . . . . . . & - & 2.4 \\
\hline Citrate de caleium $\ldots \ldots \ldots \ldots \ldots \ldots \ldots \ldots$ & 23.55 & 17.4 \\
\hline Citrate de magnésium $\ldots \ldots \ldots \ldots \ldots \ldots \ldots$ & 4.05 & 4.6 \\
\hline Phosphate de ealcium. & 16.32 & 5.3 \\
\hline Phosphate de magnésium . . . . . . . . . . . . . & 3.71 & 1.5 \\
\hline Chaux liée par la caséine ................. & 5.13 & 3.5 \\
\hline Total & 100.00 & 99.8 \\
\hline
\end{tabular}

\title{
LE FACTEUR C (antiscorbutique) DANS LE LAIT DE VACHE. SES RAPPORTS AVEC LA CONCENTRATION, L'HOIMOGÉNÉISATION ET LA STÉRILISATION (1)
}

\author{
par P. LAVIALLE, \\ Professeur à l'Université de Strasbourg
}

Les recherches exposées dans cette note ont eu pour but de déterminer l'influence de la concentration, de l'homogénéisation et de la stérilisation, sur le facteur antiscorbutique du lait de vache.

Choix de I'animal d'épreuve. - RANDuin L. a montré (2) que le cobaye exige, quotidiennement, le tiers ou la moitié de son poids de lait frais, pour éviter les accidents scorbutiques. Cet animal ne peut done pas servir à l'étude des aliments destinés à l'homme dont les exigences en facteur $\mathrm{C}$ sont beaucoup moindres.

Mon attention a été attirée sur le chien, dont le scorbut a fait l'objet d'un certain nombre d'observations (3) et présente des caractères rappelant assez exactement ceux du scorbut humain.

J'ai pu, à l'aide d'une alimentation abondante et normale en tous points, mais carencée complètement en facteur C, provoquer chez plusieurs chiens des accidents scorbutiques très nets: sensibilité des articulations; taches rougeâtres ou bleuâtres de la peau, au niveau des épiphyses surtout; gonflement des épiphyses; ulcérations ; tuméfaction des gencives; déchaussement des dents ; haleine fétide ; diarrhée intense et persistante ; déjections sanguinolentes; hématurie; muqueuses fortement décolorées.

Ces accidents surviennent au bout de 7 à 10 mois de régime carencé en facteur C : période d'incubation qui rappelle assez exactement celle du scorbut infantile.

(1) Extrait du Bull. Internat. de la Protection de l'enfance. No 49 , juin 1926.

(2) Randoin (Mme L.) Bull. Soc. Chim. biol. T. V. 1923.

(3) Gangee (Miss). Bull. Soc. Péd. Mars 1922, p. 107.

KarR (W. G.) Proc. Soc. Exper. Biol. and. Med., T. XVII, 1920, p. 84. 
Choix du lait. - J'ai utilisé un lait industriel réunissant les caractères que je désirais étudier : lait pur, non additionné de sucre, concentré à la moitié environ de son volume, homogénéisé et stérilisé. Les caractéristiques de ce lait sont les suivantes :

$1^{\circ}$ Lait d'herbage, provenant de vaches séjournant aux pâturages presque toute l'année et vraisemblablement très riche en facteurs accessoires divers ;

- $2^{\circ}$ Rapidité du traitement du lait aussitôt après la traite ;

$3^{\circ}$ Manipulations effectuées à l'abri de l'air;

$4^{\circ}$ Conservation du lait en boîtes presque complètement remplies et à parois opaques ;

$5^{0}$ Acidité du lait doublée par la concentration.

\section{EXPÉRIENCES}

Voici la série d'essais que j'ai institués au début de novembre 1924, et qui se sont terminés en mars 1926, soit une durée de 15 à 16 mois.

J'ai mis en expérience 5 chiens "fox " en très bonne santé. Chacun d'eux prenait à discrétion, à 18 heures, une soupe composée de pain, viande, os, sel, eau. Deux fois par semaine, la viande était remplacée par du lait (200 gr. environ par chien). L'ensemble était carencé en facteur C, par chauffage quotidien à l'autoclave à $125^{\circ}$ pendant 45 minutes.

Le matin à 8 heures, chaque chien recevait une ration de $500 \mathrm{cc}$. de lait: lait cru (ehien $\mathrm{n}^{0} 1$, témoin); lait concentré commercial surchauffé par moi à $125^{\circ}$ pendant 45 minutes (chien $n^{\circ} 2$, témoin); lait commercial non surchauffé par moi (chiens $\mathrm{n}^{\mathrm{os}} 3,4,5$ ).

Voici les résultats obtenus :

Chien $n^{\circ} 1$. - Ce chien témoin recevant, comme source unique de facteur C, 500 grammes de lait de vache cru présente, au bout de 5 mois de ce régime, une gastro-entérite grave, qui cesse dès que le lait eru est remplacé par du lait concentré commercial, reprend avec un lait cru d'une autre origine, est encore guérie par le lait concentré, récidive enfin sans guérison possible avec un second retour au lait cru.

Chien $n^{0} 2$. - Ce chien témoin recevant, comme unique source de facteur C, 500 grammes de lait concentré commercial surchauffé par moi à $125^{\circ}$ pendant 45 minutes, présente les symptômes du scorbut au bout de 9 à 10 mois.

Chiens $n^{\text {os }} 3,4,5$. - Ces trois animaux recevant, comme unique source de facteur C, 500 grammes de lait concentré commercial non surchauffé par moi, ont pu subir ce régime pendant 15 à 16 mois sans présenter aucun symptôme de scorbut.

Il semble résulter avec évidence de ces essais, que le lait utilisé (concentré, homogénéisé, stérilisé) renferme le facteur C en quantité ou qualité suffisante pour protéger contre le scorbut, pendant 16 mois, des chiens en bonne santé initiale. 


\section{Conclusions}

Le chien paraît de beaucoup préférable au cobaye pour la détermination des carences alimentaires en facteur $C$, surtout lorsque les résultats doivent tendre à éclairer l'alimentation humaine.

L'expérience sur le chien $\mathrm{n}^{\circ} 1$ confirme les observations des cliniciens relatives au danger de l'alimentation des nourrissons par le lait cru.

Les connaissances que nous possédons sur le facteur C, en particulier sur sa sensibilité à l'action de la chaleur et de l'air, et sur l'action préservatrice qu'exerce sur lui l'acidité normale du milieu, jointes à mes observations personnelles sur le lait étudié et aux résultats qui viennent d'être exposés, conduisent à proposer aux pédiâtres, pour le choix des laits à réserver aux nourrissons, les règles suivantes :

a) Laits d'herbage, riches en facteurs accessoires en général. Cette richesse étant fonction de la richesse en ces mêmes facteurs de l'alimentation des vaches laitières, il est évident qu'un lait d'herbage est plus riche qu'un lait produit au cours d'une longue stabulation hivernale. Il est vraisemblable aussi qu'un lait de cette dernière catégorie conservé, ne permettrait pas de maintenir un organisme en équilibre nutritif aussi longtemps qu'un lait d'herbage traité de même façon.

b) Préparation effectuée rapidement, aussitôt que possible après la traite, entièrement à l'abri de l'air et en s'aidant du vide qui accélère l'évaporation du lait et le désaère complètement.

c) Récipients de conservation remplis aussi complètement que possible pour éviter l'action de l'air.

d) Considérer comme favorables, en ce qui concerne la conservation du facteur C, toutes conditions (concentration en particulier) qui, sans l'intervention d'une fermentation microbienne dont les conséquences sont à éviter, peuvent augmenter l'acidité normale du milieu et protéger par là le facteur C contre les agents de destruction.

e) Jusqu'à plus ample information, eonserver le lait à l'abri de la lumière, dans des récipients à parois opaques (1).

La marge certaine de 15 à 16 mois laissée aux chiens nourris au lait concentré non sucré, permet d'envisager sans inquiétude l'alimentation des nourrissons normaux au moyen de laitss concentrés de bonne fabrication.

D'ailleurs, jamais le nourrisson ne reçoit pendant une période aussi longue une alimentation lactée absolument exclusive. A l'apparition des premières dents, on commence à varier un peu le régime, et on s'efforce d'y faire entrer, pour une part, des aliments frais tels que : purée de pommes de terre ou de légumes frais, fruits frais ou très peu-cuits, et non pas séulement des aliments vieillis. Nombre de cas de scorbut sont, en effet, observés chez des enfants soumis à un régime composé en partie de lait stérilisé et en partie d'aliments à carences multiples.

(1) Lesní et Vaghtano. Le Lait, décembre 1925. 
Mes expériences sont d'accord avec les faits enregistrés en pédiâtrie. Les eas de scorbut infantile sont rares, surtout en France. Cependant, on élève aux laits conservés un nombre considérable d'enfants qui supportent facilement le régime et prospèrent eonvenablement. Si tous ces laits étaient entièrement dépourvus de facteur $\mathrm{C}$, le scorbut infantile ne serait pas une rareté.

J'insiste, en terminant, sur le point suivant. Pour utiliser les laits conservés avec sécurité dans tous les cas, même chez les débiles et les atrophiques pour lesquels leur richesse habituelle et leur digestibilité sont si précieuses, il sufflt d'administrer régulièrement des sues de fruits frais (orange, raisin). Il suffirait de généraliser l'emploi de ces sucs, de les considérer cumme des satellites normaux de tout lait conservé ou même de tout lait bouilli, pour voir diminuer eonsidérablement, sinon disparaittre eomplètement, les cas de scorbut infantile.

\section{CONTRIBUTION A L'ÉTUdE DE LA MIYCOLOGIE DU « KAJMAK »}

(Deuxième Mémoire)

\section{Par M. le Professeur Dr Lovis GUTSCHY}

Directeur du Laboratoire de bactériologie à la Faculté d'agronomie de l'Université * à Zagreb (Yougoslavie)

\section{Autres microbes acido-présurigènes}

Outre les bacilles du Kajmak VI, on trouve dans le Kajmak encore d'autres microbes du groupe des microbes acido-présurigènes. Tous ces microbes montrent les qualités caractéristiques des microbes acidoprésurigènes, mais leurs fonctions les faisant ressembler avec le bacille VI, nous serrons brefs dans leur description. Nous nous bornerons a leurs qualités particulières:

\section{A. Le bacille de Kajmak VIII.}

Ce bacille se présente comme un court bâtonnet immobile, d'une longueur de 4-5 $\mu$, paraissant droit, mais souvent aussi quelque peu courbe. Une formation de spores n'a pas été observée. Les bacilles provenant de vieilles cultures montrent souvent un aspect granuleux. Le bacille est anaérobie facultatif. Une végétation aérobique comme chez le bacille VI, n'a pas été observée. Il prend le Gram. On a obtenu les meilleures eultures sur un milieu à l'extrait de malt (d'après MAKRINOFF) de la composition suivante :

$250 \mathrm{gr}$. de malt d'orge ont été chauffés dans un litre d'eau pendant une heure à une température de $60^{\circ} \mathrm{C}$., on filtre, on complète à 1 litre et on ajoute :

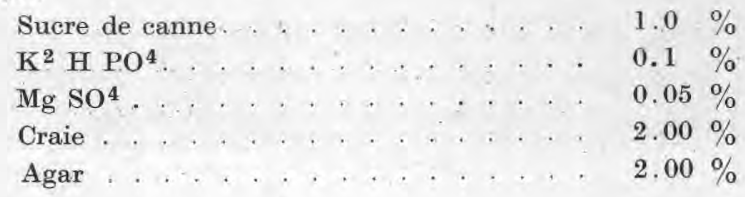

